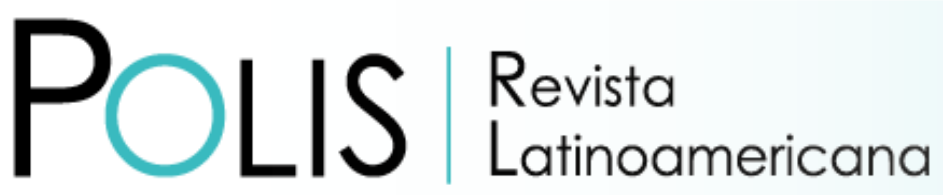

V20 | N60 | 2021

DOI: $10.32735 / \mathrm{S} 0718-6568 / 2021-\mathrm{N} 60-1595$

\title{
La autonomía de las universidades chilenas para sancionar he- chos de violencia contra las mujeres
}

\author{
Carla luspa Santelices \\ Universidad Austral de Chile, Valdivia, Chile \\ Email: carla.iuspa@uach.cl
}

Recibido:17.11.2020 | Aceptado: 19.03.2021

\begin{abstract}
Resumen: El presente trabajo analiza las consideraciones de la Corte Suprema chilena suscitadas al alero de la reciente normativa universitaria en materia de acoso sexual y otras formas de violencia en contra de las mujeres. Se sostiene que la tesis del máximo tribunal chileno (replicada por algunas Cortes de Apelaciones) adolece de una ceguera importante frente a las desigualdades de género que subyacen a los fenómenos de acoso y violencia en contra de las mujeres y que fundamentan la institucionalidad universitaria en la materia. Para ello, se analiza desde una perspectiva de género la forma de reconstruir la autonomía universitaria para sancionar dichos hechos y se contrasta con otros pronunciamientos de la Corte en que se otorga una mayor amplitud a la potestad sancionatoria de las universidades en el marco regulatorio chileno.
\end{abstract}

Palabras clave: Acoso sexual; violencia contra las mujeres; perspectiva de género; autonomía universitaria.

\section{The Autonomy of Chilean Universities to Punish Acts of Violence against Wo- men}

\begin{abstract}
This paper analyzes the considerations of the Chilean Supreme Court raised under the auspices of the recent university regulations on sexual harassment and other forms of violence against women. It is argued that the thesis upheld by the highest Chilean court suffers from a significant blindness to the gender inequalities that underlie the phenomena of harassment and violence against women and that underlie the university institutions in the matter. To do this, the way to rebuild university autonomy to sanction such acts is analyzed from a gender perspective and contrasted with other pronouncements of the Court in which greater scope is granted to the sanctioning power of universities in the Chilean regulatory framework.
\end{abstract}

Keywords: Sexual harassment; violence against women; gender perspective, university autonomy. 


\section{A autonomia das universidades chilenas para punir atos de violência con- tra as mulheres}

Resumo:Este artigo analisa as considerações da Suprema Corte do Chile levantadas sob os auspícios dos recentes regulamentos universitários sobre assédio sexual e outras formas de violência contra as mulheres. Argumenta-se que a tese defendida pela mais alta corte chilena sofre de uma significativa cegueira para as desigualdades de gênero que estão por trás dos fenômenos de assédio e violência contra as mulheres e que fundamentam a institucionalidade universitária na matéria. Por isso, analisa-se desde uma perspectiva de gênero a forma de reconstruir a autonomia universitária para sancionar tais atos e contrapõe-se a outros pronunciamentos da Corte nos quais se outorga uma maior abrangência ao poder sancionador das universidades no marco regulatório chileno.

Palavras-chave: Assédio sexual; violência contra as mulheres; perspectiva de gênero, autonomia universitária.

\section{Como citar este artículo:}

Iuspa Santelices, C. (2021). La autonomía de las universidades chilenas para sancionar hechos de violencia contra las mujeres. Polis Revista Latinoamericana, 20 (59), 174-192. doi: http://dx.doi.org/10.32735/S0718-6568/2021-N60-1595

\section{Introducción}

Las movilizaciones feministas del año 2018 tuvieron importantes repercusiones en las universidades chilenas. Entre las demandas levantadas bajo la consigna de una «educación no sexista y libre de violencia», se encontraba la exigencia de que las universidades implementaran políticas o protocolos dirigidos a la prevención, investigación y sanción de actos de sus integrantes que puedan ser constitutivos de acoso sexual o de otras formas de violencia en contra de las mujeres. En retrospectiva, parece sensato reconocer que el corazón de dichas demandas se encuentra en la constatación de tres circunstancias relevantes para las mujeres chilenas:

En primer lugar, en la constatación de que los fenómenos de violencia de género en las universidades han quedado históricamente en la más absoluta impunidad, debido a la falta generalizada de políticas dirigidas a su prevención, investigación y eventual sanción. A nivel nacional, existen antecedentes de que en 2015 no existían instrumentos universitarios dirigidos a tal fin o al menos no eran accesibles (Nash, 2015); mientras que en 2017 solo 7 universidades chilenas contaban con alguna normativa dirigida al efecto (Muñoz-García, Follegati y Jackson, 2018).

En segundo lugar, en la constatación de que los fenómenos constitutivos de acoso o violencia sexual tienen un importante componente de género, esto es, que afectan en la inmensa mayoría de los casos a las mujeres, precisamente por el hecho de serlo. En efecto, de acuerdo con las cifras publicadas por el Observatorio contra el acoso de Chile en julio de 2020, un 64\% de las mujeres encuestadas han experimentado por lo menos un tipo de acoso en el transcurso de su vida, en contraste con un $26 \%$ de hombres (p. 11). En el contexto 
educativo, el $41,4 \%$ de mujeres reconoce haber sufrido alguna situación de acoso, mientras que el porcentaje de hombres que reconocen dicha circunstancia es de un $26,4 \%$ (p. 44). En cuanto al victimario, los mismos datos muestran que en general son otros compañeros o compañeras los principales victimarios de estas conductas $(65,4 \%)$, y que en aproximadamente 9 de 10 casos en que una mujer es víctima de acoso sexual en el ámbito educativo, se reconoce a un hombre o a un grupo de hombres como victimario(s) (88,6\%) (pp. 53-55).

En tercer lugar, en la constatación de los perniciosos efectos que la violencia tiene en las mujeres que desarrollan su vida personal, laboral y/o académica en los diversos campus universitarios. Las mismas cifras del Observatorio contra el acoso en Chile muestran que 1 de cada 4 mujeres que fue víctima de acoso sexual mientras cursaba sus estudios abandonó lugares y/o situaciones comunes con su agresor (26,9\%), mientras que 1 de cada 10 mujeres asistieron a algún tipo de terapia (psicológica, psiquiátrica o alternativa) tras los hechos $(10,2 \%)$ o abandonaron definitivamente sus estudios (9,3\%) (p. 57).

A dos años de dichas movilizaciones, existen importantes avances en la materia. A la fecha, más de 40 instituciones de educación superior chilenas mantienen en sus páginas web políticas, protocolos o reglamentos dirigidos a la prevención, investigación y/o sanción del acoso sexual u otros tipos de violencia en contra de las mujeres cometidos por sus integrantes y/o en los espacios universitarios'. Además, varios planteles han avanzado en la creación de Direcciones u Oficinas dedicadas a las temáticas de género² y/o han realizado diagnósticos sobre las desigualdades de género presentes en sus contextos ${ }^{3}$.

Sin embargo, dichos avances no han estado exentos de polémicas a nivel nacional. Una de ellas es la judicialización de dichas instancias por medio de recursos de protección, a través de los que los investigados o sancionados por infringir dicha normativa reclaman ante las Cortes de Apelaciones el restablecimiento del imperio del derecho por haberse vulnerado durante la investigación y/o con ocasión de la sanción diversos derechos que la Constitución Política de la República (en adelante, Constitución) les asegura. Como consecuencia de ello, las Cortes han tenido la oportunidad de referirse a esta nueva institucionalidad, generando una serie de problemas para las respectivas investigaciones y la aplicación de sanciones a quienes han cometido hechos constitutivos de violencia en contra de las mujeres en el contexto universitario.

El presente trabajo tiene como objetivo analizar las consideraciones de la Corte Suprema chilena sobre esta institucionalidad, que han sido replicadas por algunas Cortes de Apelaciones a nivel nacional. Como se verá, la tesis y argumentos sostenidos razonan principalmente sobre el contenido y alcance de la autonomía que tienen las universidades

\footnotetext{
1 Es importante destacar que algunas Universidades chilenas contaban con dicha normativa con anterioridad a las movilizaciones del 2018 (Universidad de Chile, la Universidad Austral de Chile y Pontificia Universidad Católica de Valparaíso).

2 Universidad de Chile, Universidad de Concepción, Universidad de Santiago de Chile, Universidad de Los Lagos, Universidad Diego Portales y Universidad de Taparacá.

3 Universidad Austral de Chile desde 2018, Universidad de Valparaíso desde 2018, Universidad de Los Lagos en 2019 y Universidad de Concepción en 2018.
} 
chilenas para investigar y sancionar hechos constitutivos de violencia en contra de las mujeres. Como argumentaré en lo sucesivo, las consideraciones contenidas en dichos fallos adolecen de una ceguera importante frente a las desigualdades de género que subyacen a los fenómenos de violencia sancionados por las universidades, lo que contrasta con otros pronunciamientos en que la Corte Suprema configura la autonomía universitaria de una manera mucho más amplia. Ello implica un importante retroceso en los avances que se habían producido en la materia, por cuanto vuelve a razonar sobre la base de que la violencia en contra de las mujeres es un asunto estrictamente privado.

Para sostener lo anterior, realizaré el siguiente recorrido: en primer lugar, me referiré a la institucionalidad universitaria en materia de acoso sexual y otras formas de violencia en contra de las mujeres; en segundo lugar, describiré brevemente la tesis y argumentos sostenidos por la Corte Suprema en el fallo a comentar, que luego han sido reiterados por algunas Cortes de Apelaciones a nivel nacional; en tercer lugar, esbozaré dos críticas que estimo que merecen dichas consideraciones desde una perspectiva de género, contrastando dichas decisiones con otros pronunciamiento que otorgan un margen mucho más amplio a las universidades para investigar y sancionar a sus integrantes por conductas que infringen normativa universitaria; en cuarto lugar, realizaré algunas reflexiones finales que estimo relevantes.

\section{El acoso sexual y la violencia en contra de las mujeres en las universidades chilenas}

La violencia en contra de las mujeres ${ }^{4}$, en sus diversas manifestaciones, es un fenómeno que excede con creces el ámbito de la vida universitaria. En efecto, la Convención Interamericana para prevenir, sancionar y erradicar la violencia en contra de la mujer o Convención Belém Do Pará (en adelante, CBDP) la define en su artículo 1 como «cualquier acción o conducta, basada en su género, que cause muerte, daño o sufrimiento físico, sexual o psicológico a la mujer, tanto en el ámbito público como en el privado». Por su parte, el artículo 2 de la CBDP establece que:

«Se entenderá que violencia contra la mujer incluye la violencia física, sexual y psicológica:

a. que tenga lugar dentro de la familia o unidad doméstica o en cualquier otra relación interpersonal, ya sea que el agresor comparta o haya compartido el mismo domicilio que la mujer, y que comprende, entre otros, violación, maltrato y abuso sexual;

b. que tenga lugar en la comunidad y sea perpetrada por cualquier persona y que comprende, entre otros, violación, abuso sexual, tortura, trata de personas, prostitución forzada, secuestro y acoso sexual en el lugar de trabajo, así como en instituciones educativas, establecimientos de salud o cualquier otro lugar, y

c. que sea perpetrada o tolerada por el Estado o sus agentes, donde quiera que ocurra».

4 En este trabajo utilizo indistintamente «violencia en contra de las mujeres» y «violencia de género». 
Al respecto, es importante destacar que el concepto de violencia en contra de la mujer establecido en la CBDP no se restringe ni en cuanto al sujeto activo (puede ser ejercida por un particular o por el Estado), ni en cuanto a la naturaleza de la violencia (incluye violencia física, sexual y psicológica), ni en cuanto a la relación existente entre sujeto activo y pasivo de la violencia (familiar, laboral, afectiva o ninguna), ni en cuanto al lugar en el que se ejerce la misma (ámbito público o privado). Asimismo, para los efectos de este trabajo, importa destacar que dicho concepto menciona expresamente el acoso sexual y la violencia en la pareja como manifestaciones de este tipo de violencia. En este sentido, se trata de una definición amplia de violencia en contra de la mujer.

Esta definición resulta pertinente para el derecho chileno por dos razones. En primer lugar, porque la CBDP fue ratificada por Chile el año 1988 y, en virtud del artículo 5 inciso 2 de la Constitución, los órganos del Estado tienen el deber de respetar y promover los derechos emanados no solo del texto de la Constitución chilena, sino también aquellos provenientes de los tratados internacionales de derechos humanos ratificados por Chile y que se encuentren vigentes. En segundo lugar, porque nuestro ordenamiento jurídico no contempla una definición -ni constitucional ni legal-sobre qué debe entenderse por violencia en contra de las mujeres 5 . En este entendido, la definición de la CBDP viene a llenar el vacío de la normativa interna en materia de qué debemos entender por violencia en contra de las mujeres.

A la CBDP se suman otros cuerpos normativos internacionales relevantes para nuestro país que regulan cuestiones relativas a la situación de desigualdad de las mujeres, como la Convención para la erradicación de todas las formas de discriminación en contra de la mujer (CEDAW, 1979), el Consenso de Montevideo sobre Población y Desarrollo (2013); el Quinto Objetivo de Desarrollo Sostenible (2015), entre otros. Como se ha reconocido, todos ellos:

«convergen en el resguardo de los derechos frente a la violencia y la discriminación en las esferas de la educación y del trabajo, constatando así la necesidad y el mandato de prevenir, atender, sancionar y reparar situaciones de acoso sexual que se viven en el mundo educativo, lo que incluye aquellas que ocurren al interior de las instituciones de educación superior» (MINEDUC, s/f, p. 11).

Si bien el derecho chileno contempla alguna normativa relativa a fenómenos de violencia en contra de las mujeres, la cobertura de dicha regulación es sumamente restringida. En este sentido, o se penalizan ciertas conductas consideradas históricamente como las más graves (típicamente a través de crímenes o delitos previstos en el Código Penal) o se regulan algunos de estos fenómenos en atención a la relación conyugal o convivencial que existe o ha existido entre víctima y agresor (violencia intrafamiliar) o a los espacios en que se verifica la violencia (el espacio público6, el ámbito laboral7, el ámbito deportivo8, etc.). Dichos cuerpos normativos no son suficientes para subsanar la falta de una ley de

\footnotetext{
5 Por el contrario, la Ley N²0.066 solo prevé una definición de «violencia intrafamiliar» (artículo 5) y no de violencia de género o en contra de las mujeres.

6 Recientemente promulgada Ley $\mathrm{N}^{\circ} 21.153$ dirigida a tipificar el delito de acoso sexual en espacios públicos.

7 Ley №20.005 que tipifica y sanciona el acoso laboral y que se hace aplicable a las relaciones dadas en el marco del Estatuto Administrativo (artículo 84).

8 Ley N²1.197 que Establece el deber de contar con un protocolo contra el acoso sexual, abuso sexual, discriminación y maltrato en la actividad deportiva nacional.
} 
protección integral contra la violencia de género, que regule la prevención, investigación y sanción efectiva de dichos fenómenos, como ocurre por ejemplo en el caso de España con la Ley Orgánica 1/2004 de 28 de diciembre de 2004 de Medidas de protección integral contra la violencia de género?.

Por otro lado, en muchísimas ocasiones, la institucionalidad nacional que interviene en la aplicación de la normativa existente es insuficiente para atender el fenómeno de la violencia en contra de las mujeres, lo que ha generado un clima de desconfianza importante frente al sistema. La deficiente capacitación de funcionarios y funcionarias públicas en materia de igualdad género, la poca destinación de recursos materiales y humanos para la atención especializada de tales causas, la masculinización de los espacios de poder y decisión sobre estos hechos y, en general, los estereotipos asociados a lo femenino resultan ser, en muchas ocasiones, barreras infranqueables para el acceso de las mujeres víctimas de violencia a la justicia chilena.

En cuanto a los estereotipos de género, en muchas ocasiones las conductas desplegadas por los distintos intervinientes de los mecanismos de prevención, denuncia, investigación y sanción de hechos de violencia en contra de las mujeres resultan sumamente revictimizantes para quienes denuncian. El fenómeno de la revictimización o victimización secundaria implica que la persona que ha sufrido un hecho de violencia vuelva a experimentar nuevamente la experiencia traumática que implicaron los hechos, principalmente a raíz de comentarios o juicios de valor emitidos por quienes reciben y tramitan las denuncias y que se refieren al comportamiento, costumbres o reacciones de las mujeres víctimas frente a los hechos. Dichos comentarios normalmente funcionan restándole valor a las denuncias o cuestionando la adecuación del comportamiento de la denunciante a un modelo de mujer víctima sumamente estereotipado: las víctimas lloran, no vuelven a vivir con sus agresores, piden auxilio, relatan lo sucedido a sus cercanos, denuncian de inmediato los hechos de violencia, entre otros.

La descripción de este escenario es suficiente para reconocer, como lo han hecho organismos internacionales de derechos humanos (en adelante, DDHH) ${ }^{10}$, que el Estado chileno incumple sus deberes de «condenar todas las formas de violencia contra la mujer» y «adoptar, por todos los medios apropiados y sin dilaciones, políticas orientadas a prevenir, sancionar y erradicar dicha violencia», contemplados en el artículo 7 de la CBDP.

Frente a estas insuficiencias, las mujeres víctimas de estos fenómenos históricamente han debido abandonar sus espacios educativos, laborales o personales. Sin embargo, desde hace algunos años las demandas por espacios libres de violencia en contra de las mujeres se han convertido en centrales en las agendas de diversos movimientos sociales. En el ámbito específico de la educación superior, las demandas del ya mencionado «mayo

9 Actualmente existe un Proyecto de Ley sobre el Derecho de las mujeres a vivir una vida libre de violencia (Boletín №11077-07).

10 Entre ellos, Comité CEDAW; el Mecanismo de seguimiento de la Convención Belém do Pará (MESECVI); la Comisión Interamericana de DDHH; la Relatoría Especial sobre la violencia contra la mujer, sus causas y consecuencias de la ONU. 
feminista» se han interpretado como parte de aquellas que pretenden la modernización de los gobiernos universitarios a través de una ampliación de la participación estudiantil en las decisiones de los planteles (Sánchez, 2018). En efecto, el gobierno universitario chileno, habitualmente calificado como «tradicional» porque el poder y su representación recae en forma principal en el estamento académico (normalmente de profesores y profesoras titulares, quienes eligen o designan mediante diversas modalidades su planta directiva y órganos de gobierno interior) (Torres, 2016), paulatinamente y sobre todo a partir de la Ley №20.843 de 2015, ha ampliado la participación a estamentos no académicos en la elección y/o conformación de órganos de gobierno universitario (Ganga y Viancos, 2018), lo que ha permitido incorporar demandas a las agendas universitarias sobre fenómenos que son puestos de relieve (al menos inicialmente) por el estamento estudiantil, como el caso de la violencia sexual y/o en la pareja entre compañeros o compañeras ${ }^{11}$. Estas demandas y su acogida por los gobiernos universitarios, ha llevado a que las universidades adquieran un papel protagónico en la regulación y respuestas que pueden entregar a las mujeres que integran sus comunidades o concurren a sus instalaciones y que son víctimas de dichos fenómenos.

\section{La judicialización del conflicto por la vía del recurso de protección}

La vigencia de estos mecanismos y su efectiva implementación en las universidades no han estado exentas de polémicas a nivel nacional. Entre ellas, se encuentra la judicialización de tales instancias por medio de recursos de protección, a través de los cuales quienes han sido investigados o sancionados por las universidades por conductas de violencia en contra de las mujeres solicitan a las Cortes de Apelaciones el restablecimiento del imperio del derecho por haberse vulnerado sus DDHH. En esta materia ha resultado central el contenido, alcances y límites de la autonomía universitaria (en adelante, AU) en el derecho chileno.

Debido a su reciente aparición y a la inestabilidad de los pronunciamientos por vía de recursos de protección, resulta imposible identificar líneas jurisprudenciales claras o uniformes que permitan guiar el trabajo de las universidades y de los implicados e implicadas en la puesta en marcha de dicha institucionalidad. Sin perjuicio de ello, resulta particularmente ilustrativa la sentencia de la Corte Suprema Rol N5453-2019, de fecha 01 de julio de 2019, que acogió un recurso de protección interpuesto por un estudiante de la Universidad Austral de Chile (en adelante, UACh) que había sido rechazado por la Corte de Apelaciones de Valdivia.

Este fallo resulta relevante por tres motivos. Primero, porque fue el primer caso en que la Corte Suprema (en adelante, Corte) se pronunció directamente sobre los alcances de la AU en el ámbito de la investigación y sanción de actos de acoso y violencia en contra de las mujeres. Segundo, porque la tesis sostenida por la Corte ha sido replicada por algunas Cortes de Apelaciones a nivel nacional y en otros fallos de la Corte Suprema. Tercero, porque el razonamiento adoptado por la Corte tiene problemáticas consecuencias en los DDHH de las mujeres víctimas de acoso y violencia en las universidades chilenas, ya que la restricción

11 Para caracterización del sistema de gobierno universitario chileno en términos comparados, su evolución y crítica, se puede recurrir a la doctrina nacional que ha trabajado extensamente la materia (Ganga, 2005; Brunner, 2008; Rodríguez, 2012; Virgili, Ganga y Figueroa, 2015; Ganga y Viancos, 2018). 
de la AU para investigar y sancionar tiene como consecuencia que estos hechos vuelvan a la impunidad, quedando sus víctimas nuevamente desprotegidas en sus espacios de trabajo o estudio.

\section{La tesis de la Corte Suprema}

A continuación, el análisis se centra en el fallo ya individualizado de la Corte Suprema (Rol №5453-2019), debido a que las otras sentencias revisadas se limitan a reiterar sus consideraciones ${ }^{12}$. A continuación, reconstruyo brevemente la tesis.

Lo impugnado y ordenado dejar sin efecto en tal sede fue una resolución emanada de la Comisión para la intervención en situaciones de acoso, violencia y discriminación entre estudiantes de la UACh, por la que se sancionaba a un estudiante por haber incurrido en actos de violencia de género y acoso psicológico en contra de su expareja, mujer y estudiante de la misma carrera. Resulta importante destacar que los hechos, constitutivos de lo que coloquialmente se denomina «violencia en el pololeo», habían ocurrido fuera de las instalaciones y actividades universitarias.

En su razonamiento, la Corte invoca la Ley N²1.091 sobre Educación Superior para sostener la ilegalidad del actuar del plantel universitario. La Corte reconoce que la dictación y aplicación del Reglamento de la UACh es una manifestación de la autonomía de que gozan las universidades en virtud de dicha ley, en cuanto les permite ejercer una potestad disciplinaria independiente. Acto seguido, la Corte reconoce que la citada ley delinea la extensión que puede tener tal potestad disciplinaria, y la confina «en lo sustantivo a los fines y proyectos institucionales» del plantel (considerando sexto). En tal sentido, reprocha a la universidad haberse arrogado la competencia para conocer de hechos ocurridos fuera de las instalaciones y actividades universitarias, ya que aunque el Reglamento contempla tal posibilidad, ella debe interpretarse en el sentido de que no basta una mera conexión personal de las y los involucrados para proceder a la investigación y sanción de los hechos denunciados, sino que resulta necesario que exista una vinculación objetiva de los hechos con el plantel, determinada por el lugar (recintos universitarios) o la actividad (actividades universitarias) (considerando séptimo).

En cuanto a la vulneración de los derechos, la Corte estimó que se vulneró el derecho a la igualdad del estudiante recurrente, porque se le hizo acreedor de una consecuencia jurídica que no resulta aplicable a los hechos en que se le dio por partícipe; así como el derecho a no ser juzgado por comisiones especiales, porque resultó sancionado, incluso con medidas que sobrepasan los márgenes académicos, por hechos propios de la competencia común de los tribunales establecidos por ley, con lo cual la UACh se constituyó en una comisión especial que carece de origen legal (considerando décimo primero). Sobre este

12 A modo ejemplar, sentencia Rol N079.473-2020 de la Corte Suprema; Rol Nº1480-2019 de la Corte de Apelaciones de Valdivia; Rol N¹2.434-2020 de la Corte de Apelaciones de Valparaíso. 
último punto, la Corte sostuvo que la UACh no fundamentó cómo se satisfacen las exigencias del Reglamento para ejercer las atribuciones fuera del recinto universitario y en el entorno de una relación de pareja, resultando arbitraria su decisión (considerando noveno).

\section{Dos problemas del fallo}

\section{Comprensión limitada de la autonomía universitaria}

Para fundar la tesis de los límites a la AU, la Corte invoca el artículo 2 letra a) de la Ley №21.091 sobre Educación Superior, que la define como «la potestad para determinar y conducir sus fines y proyectos institucionales en la dimensión académica, económica y administrativa, dentro del marco establecido por la Constitución y la ley». A partir de la mención que dicha ley hace de los «fines y proyectos institucionales» de las universidades, la Corte interpreta que esta constituye no solo contenido de la $\mathrm{AU}$, sino también su límite. Luego, usa dicho límite para aplicarlo a la potestad sancionatoria de la universidad: solo se pueden investigar y sancionar hechos que hayan ocurrido dentro de las instalaciones o en el contexto de actividades universitarias. Esta comprensión, sin embargo, aparece como extremadamente limitada, particularmente si se interpreta a la luz de las demás disposiciones del marco normativo que rige la AU y si se compara con la delimitación que la doctrina chilena y la misma Corte ha hecho de esta para investigar y sancionar otro tipo de hechos.

En cuanto al marco normativo de la $\mathrm{AU}$, existen varias razones para rechazar la interpretación que la Corte entrega al artículo 2 letra a) de la Ley №21.091. En primer lugar, la Corte no entrega razones por las que el artículo mencionado deba interpretarse asimilando los «fines y proyectos institucionales» a una exigencia de vinculación objetiva de los hechos con el plantel para poder ejercer la potestad disciplinaria. Esta exigencia es creada sin entregar razones interpretativas o de otra naturaleza para su mantención.

En segundo lugar, porque los «fines y proyectos institucionales» de una universidad deben ser consonantes con la promoción y protección de los DDHH de sus integrantes. En el ámbito normativo y disciplinario, esto se traduce (entre otras cosas) en la obligación de las universidades de dictar y aplicar normas destinadas a investigar y sancionar hechos que atenten contra tales garantías, de forma de establecer las responsabilidades disciplinarias correspondientes por contradecir tales fines y proyectos. Si la promoción y protección de los DDHH de los miembros de la comunidad es un objetivo fundamental de la AU, la dictación y aplicación de normas destinadas a la prevención, investigación y sanción de conductas que atenten contra tales derechos no es solo una posibilidad entre varias, sino una exigencia para las universidades chilenas.

En atención a lo anterior, llama la atención que la Corte Suprema presuponga que la AU implica una absoluta libertad para las universidades para determinar su potestad sancionatoria de la forma que estimen pertinente, siempre y cuando se concentren en la investigación y sanción de hechos que ocurren dentro de las instalaciones o en el contexto de 
actividades universitarias (que tienen una «vinculación objetiva» con el plantel). Resulta extraño porque, como veremos a continuación, la normativa y doctrina relevante razonan sobre la base de que el contenido y los límites de la AU están, en primer lugar, dirigidos a la protección de los DDHH de quienes integran los planteles universitarios.

Existen varias razones para apoyar una interpretación diferente a la sostenida por la Corte (y replicada por ciertas Cortes de Apelaciones), a saber:

Primero: Una interpretación sistemática de la AU prevista en la letra a) de dicho artículo exige tener en cuenta los demás principios que articulan el sistema chileno de educación superior. El mismo artículo 2 de la Ley № 21.091 enumera, entre los principios del sistema de educación superior, tanto el de diversidad de proyectos educativos institucionales (letra d) como el de respeto y promoción de los DDHH (letra i). Estos principios dan cuenta de que dicha ley admite la pluralidad de visiones y valores sobre la sociedad, así como las formas de transmitirlos, teniendo en cuenta como principio fundamental el respeto y la promoción por los DDHH de los miembros de la comunidad, en todos los ámbitos de la vida universitaria, mencionando expresamente que el acoso sexual y laboral, así como la discriminación arbitraria, son contrarias a los DDHH y dignidad de las personas. Dichos preceptos establecen textualmente:

«d) Diversidad de proyectos educativos institucionales [...] que se expresa en la pluralidad de visiones y valores sobre la sociedad y las formas de búsqueda del conocimiento y su transmisión a los estudiantes y a la sociedad, incluyendo el respeto a los valores democráticos, la no discriminación arbitraria y la interculturalidad. [...]

i) Respeto y promoción de los derechos humanos [...] tanto en sus propuestas formativas, de desarrollo organizacional, como también en las relaciones de trabajo y aprendizaje. El acoso sexual y laboral, así como la discriminación arbitraria, atentan contra los derechos humanos y la dignidad de las personası (destacados propios).

Segundo: La obligación de las universidades de adoptar medidas de investigación y sanción del acoso y violencia en contra de las mujeres que promuevan el respeto y promoción de los DDHH de los y las integrantes de la comunidad universitaria es coherente con el resto de los cuerpos normativos que regulan el sistema educacional chileno.

La Ley N²1.094 sobre Universidades Estatales establece, en su artículo 49, que las prohibiciones para el personal de las Universidades estatales «relativas a actos atentatorios a la dignidad de los demás funcionarios, incluido el acoso sexual, el acoso laboral y la discriminación arbitraria» incluirán aquellos casos en que dichas conductas resultan "atentatorias de la dignidad de estudiantes, y de toda persona vinculada, de cualquier forma, a las actividades de la respectiva institución» (destacados propios). El artículo prevé derechamente la obligación de las universidades de establecer mecanismos para determinar la responsabilidad administrativa por este tipo de casos, incluyendo la necesidad de protección de los derechos y garantías procesales de las víctimas y de quienes sean investigados por dichos hechos. 
La Ley N²0.370 General de Educación contiene varios preceptos que también resultan relevantes para estos efectos. Por un lado, prescribe que la educación es un proceso que «se enmarca en el respeto y valoración de los derechos humanos» (artículo 2); que debe propender a asegurar que todas y todos los estudiantes «independientemente de sus condiciones y circunstancias, alcancen los objetivos generales y los estándares de aprendizaje» (artículo 3 letra b); que «tengan las mismas oportunidades de recibir una educación de calidad» (artículo 3 letra c); y que se «respete su integridad física, y moral, no pudiendo ser objeto de tratos vejatorios o degradantes» (artículo 10 letra a). Asimismo, el artículo 16 b) contiene una definición amplia de acoso escolar, que no se restringe en cuanto al lugar donde ocurrieron los hechos y prevé una obligación de los establecimientos educacionales de contar con mecanismos idóneos para la prevención y tratamiento del acoso escolar de cualquier tipo.

Respecto de estos últimos preceptos, cabe recalcar que las consecuencias de la violencia en contra de las mujeres en comunidades como las universitarias son múltiples. En efecto, se ha reconocido que el acoso y la violencia «afectan las oportunidades de educación de las y los estudiantes, transgreden el derecho a trabajar en un lugar propicio para el cuerpo académico y administrativo, y perturban el desarrollo personal de las víctimas» (MINEDUC, s/f, p. 16), pudiendo expresarse en consecuencias tanto a nivel subjetivo/emocional de las víctimas, en sus condiciones y/o desempeño y también a nivel institucional, configurando un clima intimidante, incómodo e inseguro tanto para las víctimas como para toda la comunidad universitaria (MINEDUC, s/f, pp. 17 y ss.). Dicho reconocimiento implica reforzar la obligación de las instituciones educativas de asegurar a todos y todas igualdad de condiciones en los ambientes educativos.

Al respecto, en las Sugerencias para la elaboración de Protocolos contra el acoso sexual en la Educación Superior elaboradas por el Ministerio de Educación chileno se reconoce que:

«la LGE establece la necesidad de que las instituciones educacionales velen para que sus cuerpos académicos y administrativos y sus estudiantes se relacionen de manera respetuosa, custodiando los derechos y la integridad física de cada integrante, y asegurando que cada estudiante acceda a una educación sin interrupciones o limitaciones provocadas por hechos violentos o amenazas que la desalienten, entorpezcan o imposibiliten» (MINEDUC, s/f, p. 9).

En la misma dirección han avanzado diversas instituciones públicas y privadas. En efecto, en la actualidad muchos organismos públicos cuentan con oficinas, instructivos o protocolos para prevenir, denunciar y/o tratar las denuncias por acoso sexual. Este es el caso de algunos servicios públicos ${ }^{13}$, que han adoptado protocolos fundamentalmente a raíz del Instructivo Presidencial $N^{\circ} 006$ de 23 de marzo de 2018 que busca «Promover y garantizar igualdad de derechos entre hombres y mujeres y elevar las exigencias de las disposiciones en materia

13 Resolución Exenta №405, de 21 de marzo de 2019 que Aprueba el procedimiento de denuncia, investigación y sanción del maltrato, acoso laboral y sexual del Servicio Nacional del Patrimonio Cultural; Protocolo de prevención, acogida, denuncia, investigación y sanción del maltrato laboral, acoso laboral y acoso sexual del Servicio de Salud Metropolitano Central (2019). 
de prevención, denuncia, investigación y sanción del maltrato, acoso laboral y sexual en la Administración Central del Estado». Otros organismos que cuentan con normativa de este tipo sonel Poder Judicial ${ }^{14}$, la Cámara de Diputados ${ }^{15}$, elSenado ${ }^{16}$, las Fuerzas Armadas ${ }^{17}$, entre otros.

En el ámbito privado, actuamente nuestro país cuenta con la Norma chilena №3262-2012 de «Sistemas de Gestión. Gestión de igualdad de género y conciliación de la vida laboral, familiar y personal» elaborada por el Instituto de Normalización, a petición del Servicio Nacional de la Mujer y Equidad de Género, y que da un «marco para que todas aquellas organizaciones laborales, académicas, gremiales, sindicales, entre otras, puedan avanzar en la implementación de procesos que contribuyan a visibilizar y reducir las desigualdades de género que se expresan en sus prácticas cotidianas» (MINSAL, 2018, p. 40). En esta línea han avanzado también partidos políticos, organizaciones deportivas y de otra índole.

Tercero: La doctrina nacional ha realizado reconstrucciones de la AU en términos del reconocimiento de los derechos fundamentales de quienes conforman las diversas casas de estudio a nivel nacional. En efecto, Jaime Bassa y Bruno Aste reconocen que «[d]esde una perspectiva constitucional, la AU se articula con una serie de derechos que contribuyen a darle contenido» (Bassa y Aste, 2019, p. 198). En su trabajo, reconstruyen la AU desde un enfoque de DDHH, reconociendo que su fundamento, contenido y límites vienen impuestos por valores y derechos constitucionales como el principio democrático, la fé pública, la consecución del bien común, el derecho a la educación, la libertad de enseñanza, entre otros (Bassa y Aste, 2019).

Los insumos legislativos y doctrinales anteriores permiten plantear que los «fines y proyectos institucionales» de las universidades deben tener como objetivo y límite la promoción y respeto de DDHH de sus miembros y, en particular, la protección de la dignidad e igualdad de oportunidades entre hombres y mujeres en el ámbito académico y laboral. Además, como hemos visto, dicho marco normativo reconoce el acoso sexual, el acoso laboral, la discriminación arbitraria y todo trato vejatorio como contrario a dichos DDHH y como conductas que las instituciones de educación superior deben comprometerse a prevenir, investigar y sancionar.

Ahora bien, ¿̇qué relación tiene este enfoque de DDHH con la institucionalidad universitaria en materia de acoso y violencia en contra de las mujeres? Como se sostuvo, dicha institucionalidad tiene como vocación subsanar, al menos en parte, las deficiencias del ordenamiento jurídico chileno en cuanto a la protección efectiva e integral de las mujeres víctimas de violencia. En relación con ello, la vocación de tal normativa es esencialmente

14 Acta №103-2018 que contiene procedimiento de actuación para la prevención, denuncia y tratamiento del acoso sexual en el Poder Judicial.

15 Protocolo de Prevención y Sanción del acoso sexual en la Cámara de Diputados, de 16 de enero de 2019.

16 Resolución interna NP-206-2018 que aprueba Protocolo para la prevención, investigación y sanción del acoso sexual, de 12 de diciembre de 2018.

17 Resolución Exenta N6800/76, del 12 de marzo de 2019 del Ministerio de Defensa que Aprueba protocolo conjunto de denuncias de acoso sexual o laboral en las Fuerzas Armadas. 
una de protección y promoción de los DDHH de los integrantes de la comunidad universitaria, sobre todo de quienes han sido históricamente vulneradas en ellos: las mujeres víctimas de violencia.

Tal objetivo está plasmado en varios de los cuerpos normativos de las universidades, en los que se invocan DDHH como: «el derecho al trato igualitario y a la no discriminación; la salvaguarda de la dignidad; el derecho a la integridad física y psíquica, la protección a la intimidad; y el derecho a una educación libre de violencia» (Política de prevención y sanción del acoso, la violencia y la discriminación en la Comunidad Universitaria UACh) que se verían vulnerados por los hechos de violencia en contra de las mujeres. En relación con ello, la normativa establece no solo normas de investigación y sanción (clásicamente «protección» de los DDHH), sino también «medidas de formación, información, difusión y sensibilización para prevenir» tales fenómenos («promoción» de tales DDHH). Todo lo anterior salvaguardando «los derechos de las y los afectados, en el necesario contexto de prudencia, proporcionalidad y confidencialidad, y velando en todo caso por las garantías de la presunción de inocencia y del derecho a la educación de las personas involucradas» (Política de prevención y sanción del AVD en la Comunidad Universitaria UACh), esto es, dando cabida a los DDHH de los integrantes de la comunidad que sean denunciados.

Lo mismo ocurre con la Universidad de Valparaíso. En efecto, el artículo 1 del Reglamento sobre normas de conducta, criterios y protocolos de actuación para prevenir y enfrentar situaciones de acoso u hostigamiento sexual o sexista, establece que las conductas allí tipificadas «Vulneran la dignidad y los derechos básicos de las personas, tales como su derecho a un trato igualitario, a la integridad física y psíquica, a la protección de su intimidad y/o a recibir una educación libre de violencia, entre otros».

De acuerdo con lo anterior, la vocación de la institucionalidad universitaria en la materia es la protección y promoción de los DDHH de las mujeres víctimas de tales fenómenos, en un contexto de igual respeto por los DDHH de quienes resulten investigados, todo al alero de la AU reconocida por las leyes chilenas. Este análisis es completamente ajeno al razonamiento de la Corte, que se concentra en crear un límite a la AU no contemplado en la ley y vulnerar el resto de normativa que exige que las decisiones de las instituciones educativas se funden en la promoción y protección de los DDHH de quienes las integran.

\section{Falta de perspectiva de género}

Una vez establecida la necesidad de interpretar el contenido y límite de la AU en consonancia con los principios de diversidad de proyectos educativos y la exigencia de promover y proteger los DDHH en virtud de ella, cabe preguntarnos si la perspectiva de DDHH es suficiente para analizar la institucionalidad universitaria en la materia. 
En este punto, resulta relevante recordar que la Corte no cuestiona la AU para establecer procedimientos de investigación y sanción de actos contrarios a dicha normativa. Tampoco analiza si, durante el procedimiento, se incumplieron las normas procedimentales adoptadas en virtud de dicha AU. Por el contrario, lo que sostiene la Corte es que dicha autonomía se encuentra limitada por el lugar o contexto en que ocurren los hechos a investigar, de manera que dicha institucionalidad debe quedar restringida, en su competencia, a lo que pase en los campus o en actividades universitarias. Todos los demás hechos que, pese a estar recogidos en los protocolos o reglamentos, ocurren en la vía pública, residencias estudiantiles, domicilios particulares, bares o fiestas, quedan completamente fuera de la competencia investigativa y sancionatoria de las universidades y normalmente en la más completa impunidad.

El problema principal de esta tesis es que solo discurre sobre la base de los derechos del investigado. La Corte no inquiere sobre los alcances que una interpretación tan restrictiva de la $\mathrm{AU}$ puede tener en los DDHH de las mujeres víctimas de violencia, que es precisamente el fundamento principal de la institucionalidad que el recurrente impugna. No se pregunta sobre la naturaleza de los fenómenos regulados ni sobre las consecuencias de la tesis que sostiene para otros DDHH como la integridad, la intimidad y el derecho a un espacio laboral o académico libre de violencia en contra de las mujeres.

Autoras feministas como Alda Facio han sostenido que todo diagnóstico o política debe ser analizada a la luz de una perspectiva de género. Este análisis «requiere que se parta de que toda acción humana impacta a hombres y mujeres de manera particular por la forma como se construyen los géneros y que, por ende, este tipo de análisis debe hacerse al estudiar cualquier fenómeno o grupo social» (Facio, 2002, p. 89), incluyendo los fenómenos de discusión, creación y aplicación del derecho. De lo contrario «lo que hacemos es utilizar la perspectiva androcéntrica que es la que pasa por una no perspectiva» (lbídem).

Esto último es precisamente lo que hace la Corte Suprema: analizar el fenómeno de la AU (específicamente la potestad normativa y disciplinaria) sin tener en cuenta las particularidades de los casos de acoso y violencia en contra de las mujeres y cómo estos influyen en los DDHH de sus víctimas. En efecto, las únicas palabras que la Corte dedica a estas razones fundantes las realiza al pasar y sin mayor profundidad: señala que la arbitrariedad de la sanción aplicada al estudiante recurrente se funda en que ha sido juzgado con sanciones que sobrepasan los márgenes académicos (considerando décimo primero), ya que se han ejercido atribuciones fuera del recinto universitario «y en el entorno de una relación de pareja» (considerando noveno).

Estas consideraciones de la Corte son significativas, porque dan cuenta de una visión androcéntrica sobre cómo debe ser la potestad normativa y disciplinaria de las universidades: centrada en lo estrictamente académico, en lo esencialmente público. La distinción artificial entre lo público y lo privado que subyace a muchas instituciones sociales y jurídicas ha sido una de las críticas clásicas de la teoría feminista no solo al derecho, sino a la forma en 
la que construimos, entendemos o hablamos de todos los aspectos de nuestra sociedad. En el caso de las universidades, la distinción entre lo público y lo privado fundamenta la idea de que «las instituciones universitarias deben encargarse exclusivamente de las cuestiones concernientes a lo académico y al conocimiento; y que la forma a partir de la cual se construye el saber es neutral» (Vázquez, 2017, p. 17).

La dicotomía entre lo público y privado ha tenido importantes implicancias en la falta de discusión de los fenómenos de violencia en contra de las mujeres en el ámbito público (relegación a la absoluta impunidad que caracteriza los espacios domésticos). Se ha sostenido que:

«la asignación diferente de esos espacios a varones y mujeres, y la circunscripción de lo político exclusivamente a la esfera pública ocupada predominantemente por los varones [...] resultaron también en la imposibilidad de poner en cuestión (hasta no hace mucho) en el espacio público y político asuntos relativos a la sexualidad y (no) reproducción de las personas» (Brown, 2014, pp. 124-125).

Desde una perspectiva de género, el análisis de los fenómenos de violencia en contra de las mujeres exige preguntarse dónde, cómo y por qué ocurren tales hechos. Ignorar que la mayoría de los actos de violencia en contra de las mujeres no ocurren en la esfera pública, sino en espacios esencialmente privados como la familia o las relaciones de pareja, es una visión sesgada de cómo ocurren los fenómenos de violencia en contra de las mujeres en una sociedad patriarcal. En efecto, tal y como recuerda Facio «muchas de las violaciones a los derechos de las mujeres suceden en la esfera privada y todas sabemos que 'los trapos sucios se lavan en casa' y no se ventilan en público» (Facio, 2002, p. 92).

Esto es precisamente lo que hace la Corte Suprema en su fallo: exigir a las universidades que se centren en investigar y sancionar los actos públicos de sus miembros, circunscribiendo al ámbito privado -y en la más amplia desprotección- aquellos fenómenos que ocurren fuera de las instalaciones o actividades organizadas o financiadas por la universidad. En este sentido, olvida que la institucionalidad que el recurrente califica de ilegal y arbitraria está fundada en una perspectiva comprehensiva de los DDHH de las mujeres: la perspectiva de género. Lo anterior no es menor por cuanto tiene como consecuencia perpetuar la vulneración histórica que se ha realizado a los DDHH de las mujeres víctimas de violencia por parte de sus parejas.

Sin perjuicio de ello, la Corte Suprema ha fallado en otras ocasiones sobre la autonomía de corporaciones públicas y privadas para investigar y sancionar por infracción de estatutos, manuales o reglamentos internos de conducta a sus miembros. Un tema relevante en tal contexto y que ha sido resuelto por la vía del recurso de protección es el de las sanciones impuestas a estudiantes de educación básica y media por conductas de acoso escolar, definido en términos amplios por el artículo 16 b) de la Ley General de Educación. En efecto, la ley no limita el acoso escolar ni en cuanto al lugar ni en cuanto al medio por el cual se verifica. Únicamente se delimita en cuanto a las personas involucradas (estudiantes) y 
al tipo de conducta (agresión u hostigamiento reiterado). Además de la definición, la ley contempla una serie de exigencias para que los establecimientos prevengan, investiguen y sancionen el acoso escolar.

La Corte Suprema ha debido conocer recursos de protección deducidos por los padres y/o madres de estudiantes que han sido sancionados por instituciones educativas por incurrir en conductas que pueden calificarse de acoso escolar. En muchas ocasiones estos actos se han verificado fuera de los establecimientos educacionales y de actividades educativas, como ocurre con el caso paradigmático del ciberacoso ${ }^{18}$.

En esta sede, a diferencia de lo que ocurrió con la tesis en materia de AU, las Cortes razonan sobre la base de la obligación de los establecimientos educacionales de promover acciones dirigidas a prevenir, investigar y sancionar todo tipo de vulneración a los DDHH de los integrantes de la comunidad educativa. Incluso, en algunos fallos, las Cortes han razonado sobre la base del interés superior del niño, niña y adolescente, sosteniendo que los hechos de acoso escolar son graves y reprochables «no solo en el plano de la convivencia sino también en un plano humano como educativo, por su naturaleza, todo establecimiento educacional debe repudiar y poner término a ellas y requerir la protección todos sus alumnos [...]» (considerando décimo tercero, Corte de Apelaciones de Coyhaique de 24 de agosto de 2012, Rol No38-2012).

Sin perjuicio de lo que ha ocurrido con los establecimientos educacionales de enseñanza básica y media, la Corte Suprema también se ha pronunciado sobre la AU para sancionar otro tipo de hechos. Un caso ilustrativo por su similitud con el comentado en esta sede es la sentencia Rol N²6.104-2019 de 17 de enero de 2020, en el que la Corte Suprema rechazó un recurso de protección interpuesto por un estudiante de la Universidad Autónoma de Chile que había sido expulsado por haber proferido comentarios ofensivos por redes sociales a personas ajenas a la universidad, relacionados con sus condiciones de salud y rasgos de identidad ${ }^{19}$.

Sostuvo, en lo esencial, que "llo controvertido en autos es el alcance que se le reconoce a la aplicación del Reglamento sobre Conducta y Convivencia Universitaria») (considerando sexto). Agrega que tal normativa entrega «claras pautas en cuanto a que el ámbito de aplicación se extiende más allá de un aspecto objetivo o físico como lo es el ámbito territorial en que se asienta la universidad apelante. Por el contrario, el reglamento atiende a un elemento subjetivo, cual es la calidad de alumno regulanı (considerando sexto).

18 En tales fallos, se han confirmado sanciones por la realización conductas de connotación sexual dentro y fuera del establecimiento escolar en contra de estudiantes (Corte de Apelaciones de Coyhaique Rol No38-2012); por amenaza a profesor fuera del establecimiento (Corte de Apelaciones Santiago, confirmada por la Corte Suprema, Rol N².259-2015); por acoso escolar dentro y fuera del establecimiento (Corte de Apelaciones de Antofagasta Rol No1431-2012).

19 La Corte Suprema revocó el fallo de la Corte de Apelaciones que había acogido el recurso (19 de agosto de 2019, causa Rol N¹202-2019). 
Una vez establecido el objetivo de la normativa, la Corte sostuvo que la conducta del recurrido es contraria tanto a los valores y principios de la universidad de trato respetuoso a las personas y respeto a la diversidad, como a la conducta esperable de un estudiante de la carrera de Medicina (considerando noveno).

Lo interesante es que si bien este fallo también inquiere sobre el contenido y los límites de la $\mathrm{AU}$ en materia de sanción por infracciones reglamentarias, la forma en que se interpreta el artículo 2 de la Ley N²1.091 es sumamente diversa. En efecto, el exigente estándar de los límites de la $\mathrm{AU}$-según el cual el estudiante sancionado fue vulnerado en sus DDHH por haber sido sancionado por hechos ocurridos fuera del plantel universitario- solo fue aplicado por la Corte para el caso de violencia de género, pero no lo fue al momento de analizar la AU para sancionar con expulsión a un estudiante que, por un canal de YouTube, profirió comentarios denigrantes a personas ajenas a la institución universitaria. Todo ello pese a que en el primer caso se trató de hechos reiterados de violencia física y psicológica y de acoso en contra de una estudiante del plantel; mientras que en el segundo caso se trató de comentarios denigrantes o discriminatorios vertidos por redes sociales en contra de personas ajenas a la comunidad universitaria.

La razón de esta falta de consistencia no se encuentra explicitada en ninguno de los dos fallos. Sin embargo, salta a la vista que solo en el primero de ellos la Corte Suprema reprocha un exceso de competencia con sanciones que exceden el ámbito académico e interfieren en la vida privada de las personas involucradas, mientras que en el segundo habilita a la universidad a sancionar a un estudiante, incluso con expulsión, por conductas realizadas por redes sociales respecto de personas ajenas a la institución.

\section{Reflexión final}

Si bien los avances en materia de prevención y sanción del acoso sexual y otras formas de violencia contra las mujeres en el contexto universitario chileno son importantes, su judicialización parece avanzar en la vía contraria a la establecida por la normativa y la doctrina nacional en materia de promoción y protección de los DDHH de quienes integran las comunidades educativas a nivel nacional.

El fallo analizado durante el presente trabajo, así como los que han replicado sus consideraciones, adolece de una importante ceguera sobre las desigualdades de género que fundamentan la normativa universitaria en la materia. Ello por cuanto ignora $u$ omite pronunciarse sobre el por qué, cómo y en qué lugares ocurren los hechos constitutivos de violencia en contra de las mujeres. Una vez que se constata que estos ocurren habitualmente en los espacios privados, la omisión de dicha consideración al establecer los contornos de la AU resulta androcéntrica, esto es, sesgada hacia el género masculino. 
Como se analizó a lo largo del presente trabajo, lo que la Corte Suprema parece reprochar a la universidad es que la sanción impuesta al estudiante haya sobrepasado los márgenes de lo público, interviniendo en hechos que considera esencialmente privados y en los que no habría un interés público o académico relevante a tutelar: la violencia en el contexto de las relaciones de pareja. El impacto de esta tesis en los DDHH de las mujeres denunciantes es evidente: las universidades deberán el alcance de su potestad sancionatoria a los pocos casos que ocurren dentro de sus instalaciones o en actividades organizadas por ellas, quedando nuevamente la gran parte de los casos de violencia en contra de las mujeres en la más absoluta desprotección.

La dirección en la que ha de avanzar la comprensión de la AU en materia de prevención y sanción del acoso y otras formas de violencia en contra de las mujeres debe integrar una perspectiva de género. Debe entender que los fenómenos que fundamentan dicha institucionalidad ocurren con mayor frecuencia a las mujeres precisamente por el hecho de serlo, en espacios (públicos) en los que el discurso dominante ha estado históricamente masculinizado.

\section{Agradecimientos}

Agradezco para la redacción del presente artículo los invaluables aportes de Yanira Zúñiga Añazco y de Katerine Fuentes Lagos, ambas docentes de la Universidad Austral de Chile.

\section{Bibliografía}

Bassa, J. y Aste, B. (2019). Autonomía Universitaria: Configuración legislativa de su contenido constitucional. Estudios Constitucionales, 17(1), 189-232.

Brown, J. (2014). Mujeres y ciudadanía en Argentina. Debates teóricos y políticos sobre derechos (no) reproductivos y sexuales (1990-2006). Buenos Aires, Argentina: Teseo.

Brunner, J. (2008). El sistema de educación superior en Chile: un enfoque de economía política comparada. Revista da Avaliação da Educação Superior (Campinas), 13(2),451-486.

Facio, A. (2002). Con los lentes de género se ve otra justicia. El otro derecho, 28, 85-102. Recuperado de https://www.te.gob.mx/genero/media/pdf/562cc59475f0864.pdf.

Ganga, F. (2005). Análisis preliminar del gobierno universitario chileno. Revista Venezolana de Gerencia (RVG). 10(30), 213-246.

Ganga, F. y Viancos, P. (2018). Reforma a la educación superior y su impacto en el sistema de gobierno universitario. El caso de Chile. Revista de Ciencias de la documentación, 4(1), 49-67.

Ministerio de Educación (s/f). Protocolos contra el acoso sexual en la Educación Superior. Sugerencias para su elaboración. Recuperado de https://www.mineduc.cl/wp-content/uploads/sites/19/2018/05/acoso-sexual-educacion-superior.pdf

Muñoz-García, A., Follegati, L. y Jackson, L. (2018). Protocolos de acoso sexual en las Universidades chilenas: una deuda pendiente. Serie de Policy Briefs. Centro de Estudios de Políticas y Prácticas en Educación, 20.

Nash, C. (2015). Respuesta Institucional ante el acoso sexual en la Universidad de Chile. Oficina de Igualdad de oportunidades de género, Universidad de Chile, 1. Recuperado de http://repositorio.uchile.cl/handle/2250/149305 
Observatorio contra el acoso Chile (2020). Radiografía del acoso sexual en chile: Primera encuesta nacional sobre acoso sexual callejero, laboral, en contexto educativo y ciberacoso. Recuperado de https://www.ocac.cl/wp-content/uploads/2020/07/Informe-encuesta-OCAC-2020.-Radiograf\%C3\%ADa-del-acoso-sexual-en-Chile.pdf

Sánchez, I. (2018). Participación y gobierno universitario. El Mercurio, Ed. 07 de agosto de 2018. Recuperado de https://redg9.cl/2018/08/07/participacion-y-gobierno-universitario/.

Torres, E. (2016). Gobierno universitario en entornos de debate social en Chile. Observaciones desde la Teoría de sistemas. Polis, Revista Latinoamericana. 15(43), 655-680.

Vázquez, V. (2017). Lo "personal es política universitaria": incumbencias de las universidades nacionales frente al acoso sexual. La Aljaba. XXI, 13-28. doi: 10.19137/la-2017-v2102

Virgili, Ganga y Figueroa (2015). Gobernanza universitaria o cogobierno: el caso de la Universidad de Concepción de Chile. Última década. 23(42). 187-216.

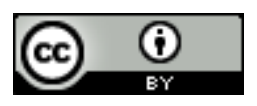

Este es un artículo de acceso abierto bajo licencia Creative Commons Reconocimiento 4.0 Internacional 